\title{
BMJ Open Bone marrow-stimulating techniques in arthroscopic rotator cuff repair: a systematic review protocol
}

\author{
Liang-Tseng Kuo, ${ }^{1,2}$ Chi-Lung Chen, ${ }^{1}$ Pei-An Yu, ${ }^{1}$ Yu-Shiun Tsai, ${ }^{2}$ Wei-Hsiu Hsu, ${ }^{1,3}$ \\ Ching-Chi Chi, ${ }^{2,3,4}$ Jae Chul Yoo ${ }^{5}$
}

To cite: Kuo L-T, Chen C-L, Yu P-A, et al. Bone marrowstimulating techniques in arthroscopic rotator cuff repair: a systematic review protocol. BMJ Open 2018;8:e022086. doi:10.1136/ bmjopen-2018-022086

- Prepublication history and additional material for this paper are available online. To view these files, please visit the journal online (http://dx.doi. org/10.1136/bmjopen-2018022086).

Received 2 February 2018

Revised 22 August 2018

Accepted 23 August 2018

Check for updates

(c) Author(s) (or their employer(s)) 2018. Re-use permitted under CC BY-NC. No commercial re-use. See rights and permissions. Published by BMJ.

${ }^{1}$ Division of Sports Medicine, Department of Orthopaedic

Surgery, Chang Gung Memorial Hospital, Chiayi, Chiayi, Taiwan ${ }^{2}$ Center of Evidence-Based Medicine, Chang Gung Memorial Hospital, Chaiyi, Chiayi, Taiwan ${ }^{3}$ College of Medicine, Chang Gung University, Taoyuan, Taiwan

${ }^{4}$ Department of Dermatology, Chang Gung Memorial Hospital, Linkou, Taoyuan, Taiwan

${ }^{5}$ Department of Orthopaedic Surgery, Samsung Medical Center, Sungkyunkwan University School of Medicine, Seoul, Korea

Correspondence to Professor Ching-Chi Chi chingchichi@gmail.com

\section{ABSTRACT}

Introduction Bone marrow-stimulating (BMS) techniques during arthroscopic rotator cuff repair surgery theoretically enhance the biological component for healing and hence improve tendon healing, but their efficacy remains unproven. The purpose of this review is to determine the effects and associated harms of BMS in arthroscopic rotator cuff repair surgery.

Methods and analysis We will perform a systematic review and meta-analysis of randomised-controlled trials (RCTs) and retrospective cohort studies (RCS) that compare outcomes following BMS use against no use of BMS during arthroscopic rotator cuff repair surgery. We will search the databases including the Cochrane Central Register of Controlled Trials, Medline and Embase, and clinical trial registries for relevant studies. We will include studies published from start of indexing until 23 August 2018. Two reviewers will independently assess the eligibility for studies. For each included trial, we will conduct duplicate independent data extraction and risk of bias assessment. We will use the Cochrane Collaboration tool to assess the risk of bias of included RCTs, while we will use the Risk Of Bias In Non-randomised Studies - of Interventions tool to evaluate the risk of bias of RCS. We will perform a random-effects meta-analysis in calculating the pooled risk estimates when appropriate. We will assess the overall quality of the data for each individual outcome using the Grading of Recommendations, Assessments, Development and Evaluation approach. The primary outcomes are tendon healing rate, overall pain and shoulder functions. The secondary outcomes are the proportion of participants with adverse events related to interventions, the range of motion and the proportion of participants with return to previous activities.

Ethics and dissemination We will report this review according to the guidance of the PRISMA statement. The results of this review will be disseminated through conference presentations and publications in peerreviewed journals.

PROSPERO registration number CRD42018087161.

\section{INTRODUCTION}

For rotator cuff tear, arthroscopic repair is a well-established treatment, with comparable outcomes with mini-open procedures. ${ }^{1} 2$ However, the healing rate of the tendon is still variable. The retear rate was reported to be

\section{Strengths and limitations of this study}

- This will be the first systematic review to provide an objective, comprehensive and systematic assessment of the use of bone marrow-stimulating techniques in arthroscopic repair surgery for patients with rotator cuff tear.

- The results of this systematic review may assist orthopaedic surgeons to make clinical decisions regarding optimal procedures in relation to arthroscopic rotator cuff tear with this patient population.

- We will assess the overall quality of the synthetic evidence by using the Grading of Recommendations, Assessment, Development and Evaluation approach.

- Conclusions and the grading of recommendations will be limited by the numbers and the quality of included studies.

$20 \%$ for small tears, and as high as $70 \%$ for large and massive tears. ${ }^{3}$ Both mechanical and biological factors contribute to tendon healing. ${ }^{4}$ With the improvement of mechanical structures including double row or transosseous suture equivalent configuration and advanced anchors with high pull-out strengths and stronger suture materials, the healing rate of the tendon has improved to be nearly $90 \% .^{5-8}$

On the other hand, investigators have suggested several augmentation procedures for enhancing biological environments in the tendon attachment site for tendon-to-bone healing. ${ }^{9}$ Platelet-rich plasma (PRP) theoretically provides exogenous growth factors, though its role in tendon healing remains controversial. From recent studies regarding the effect of PRP on rotator cuff repair, ${ }^{10-12}$ application of PRP during arthroscopic procedures may improve tendon healing rate and clinical outcomes. Bone Marrow-stimulating (BMS) techniques are the other strategy proposed to improve biologic environments of tendon-bone healing, and have been used clinically in microfractures or drilling for chondral defects ${ }^{13} 14$ and multiple 
channelling for rotator cuffs. ${ }^{15}$ BMS involve disruption of subchondral bone and subsequent migration of healing components including growth factors and mesenchymal stem cells from bone marrow cavity. ${ }^{15}$ Some authors reported the favourable outcomes of using BMS during arthroscopic rotator cuff repair procedures. ${ }^{15-17}$ Experimental studies have shown that BMS significantly increased force to failure and with thicker collagen fibres and more fibrocartilage histologically. ${ }^{18} 19$

Despite potential role as biologic repair adjunct, the disruptive behaviours of subchondral bone of BMS do damage the bony structure. It takes the risk of interfering the mechanical strength of fixation device, hence influencing the tendon healing, given the fact that these patient populations are mostly elderly with degrees of osteopenia or osteoporosis. Meanwhile, the addition of $B M S$ may increase the complexity of procedures and prolong the operation and anaesthesia time, which may be harmful to patients.

\section{Why is it important to conduct this review?}

BMS techniques are increasingly perceived as a complementary strategy during arthroscopic rotator cuff repair surgery, ${ }^{15-17}$ but their associated harms and benefits have never been systematically evaluated. No systematic review has yet focused on the benefits or harms of BMS in arthroscopic rotator cuff repair surgery.

\section{Research question}

Does the use of BMS during arthroscopic rotator cuff repair improve the tendon healing rate and clinical outcomes of surgery, compared with standard arthroscopic rotator cuff repair surgery without the additional use of BMS?

\section{METHODS AND ANALYSIS}

\section{Criteria for considering studies for this review}

\section{Type of studies}

We will include all randomised-controlled trials (RCTs) and observational studies (case-control or cohort). We will exclude studies without a control group such as case series and case reports. We will also exclude non-clinical studies such as cadaveric and animal studies.

\section{Type of participants}

We will include studies that focus on patients with full-thickness (complete) tear of the rotator cuff (mainly supraspinatus tendon) who undergo arthroscopic rotator cuff repair surgery. We will exclude studies with a primary focus on patients with other pathologies including glenohumeral osteoarthritis, irreparable rotator cuff tear, subscapularis tear, cervical neuropathy, ipsilateral upper limb injury and other diseases that may affect upper limb functions. We will exclude studies mixing patients with full-thickness tear and partial-thickness tear of the rotator cuff unless separate data for the full-thickness tear are obtained. We will exclude studies that focus on paediatric patients $(<18$ years) and animals.

\section{Type of interventions}

The interventions of interest are use of any kind of BMS techniques (microfractures, multiple channelling, multiple drilling, etc) during arthroscopic rotator cuff repair. We will include studies comparing use of any kind of BMS during arthroscopic rotator cuff repair surgery with no use of BMS during arthroscopic rotator cuff repair surgery. We will also include studies comparing using two different BMS techniques during arthroscopic rotator cuff repair surgery.

\section{Type of outcome measures}

\section{Primary outcomes}

1. Tendon structural integrity measured by MRI or CT arthrography.

A. MRI criteria by the Sugaya's classification: ${ }^{20}$

- Type I, repaired cuff of sufficient thickness with homogeneous low intensity in each image.

- Type II, sufficient thickness associated with a partial high-intensity area.

- Type III, insufficient thickness without discontinuity.

- Type IV, minor discontinuity in more than one slice, suggesting a small tear.

- Type V, major discontinuity in each image, suggesting a medium to large tear.

B. CT arthrogram criteria by the modified Boileau grading system: ${ }^{21}$

- Healing: healing and incomplete healing.

- Retears: any types of retears and new tears.

2. Overall pain level measured by Visual Analogue Scale, Verbal Rating Score, Numerical Rating Scale or other validated instruments used to assess pain.

3. Function or disability, assessed by upper limb functional measures instruments, including but not limited to:

- American Shoulder and Elbow Surgeons Shoulder Score. ${ }^{22}$

- Shoulder Pain and Disability Index. ${ }^{23}$

- University of California at Los Angeles (UCLA) Shoulder Scale. ${ }^{24}$

- Constant Score. ${ }^{25}$

- Neer rating. ${ }^{26}$

- Disability of the Arm, Shoulder and Hand Score. ${ }^{27}$

\section{Secondary outcomes}

- Proportion of participants with adverse events related to intervention (ie, fracture, the need for additional surgery).

- Functions assessed by range of motion (active and passive).

- Numbers of participants returning to previous activities (work, sport, activities of daily living, etc), including time to return.

\section{Timing}

If the outcomes are examined at multiple time points, we will extract the data at the following time points: baseline, 
postoperative (within 1 week after index surgery); shortterm follow-up (up to 3 months following intervention); intermediate follow-up (longer than 3 months and up to 6 months after index surgery); and long-term follow-up (longer than 1 year after index surgery).

\section{Search methods for identification of studies}

We will search databases including Medline, Embase, the Cochrane Central Register of Controlled Trials for relevant studies. We will include studies published from start of indexing until 23 August 2018. We will also explore trial registers including the WHO International Clinical trials Registry Platform (www.who.int/ictrp/en/) and ClinicalTrials.gov (www.clinicaltrials.gov) for any ongoing trials. We will search all databases from inception to the present without restriction of languages. See online appendix 1 for the search strategy for Medline. We will use a similar approach with keywords in searching other databases.

\section{Searching other resources}

We will look for additional relevant studies by hands-on check of the reference lists of all included studies and relevant reviews. We will contact the specialist in this field for any ongoing trials or data which are not published or reported.

\section{Data collection and analysis}

\section{Selection of studies}

At first, we will remove duplicate records of the search results. Two review authors (L-TK and C-LC) independently checked titles, abstracts and keywords from the search records to identify potentially eligible studies. Second, the same two review authors will check the full text of potentially eligible studies independently. These two review authors will reach consensus by discussion, and a third review author (C-CC) will be consulted if necessary.

\section{Data extraction and management}

We will use a standardised data extraction sheet to collect study characteristics and outcome data of included studies. One review author (L-TK) will extract the following data from the included studies, whereas the second review author (C-LC) will check the accuracy.

- Methods: publication year, study design, study centres and locations, withdrawals, and duration of study (follow-up).

- Participants: patient number, age (mean or range), sex, diagnosis of disease, disease condition (small or large tear), disease duration, diagnostic method, inclusion and exclusion criteria.

- Interventions: invention, comparison and co-interventions. We will extract the techniques of rotator cuff repair, any additional procedures and operation time.

- Outcomes: prespecified primary and secondary outcomes, time points when results were measured.

- Notes: funding of study, notable declaration of conflicts from authors.
We will report the characteristics of included studies in a table, including their level of evidence, number of patients and treatment methods.

Assessment of risk of bias in included studies

Two review authors independently assessed the risk of bias for RCTs using the Cochrane Collaboration risk of bias tool. ${ }^{28}$ We will resolve disagreements by discussion, where necessary, in consultation with the third review author (C-CC). We assess the following seven domains for risk of bias of RCTs: randomisation, allocation concealment, performance bias (blinding of patient and personnel), detection bias (blinding of outcome assessment), incomplete outcome data, selective reporting and other biases. ${ }^{29}$ We will judge the domains mentioned above as being at high risk of bias, low risk of bias or unclear risk of bias. ${ }^{28}$ For the studies with a non-RCT design, we will use the Risk Of Bias In Non-randomised Studies - of Interventions tool for assessing the risk of bias. ${ }^{30}$ We will use the Grading of Recommendations, Assessments, Development and Evaluation (GRADE) approach to access the overall quality of the data for each outcome. ${ }^{31}$

\section{Measures of treatment effect}

We will express pooled data with mean difference or standardised mean difference with $95 \%$ CI for continuous outcomes and risk ratios (RRs) with $95 \%$ CI will be used for dichotomous outcomes. When the RR is statistically significant, we will also calculate the number needed to treat to benefit, and number needed to treat to harm.

\section{Unit of analysis issues}

We will use individual participants as the unit of analysis. For studies including bilateral surgery, we will follow the analytical methods stated in section 16.3 of the Cochrane Handbook for Systematic Reviews of Interventions. ${ }^{29}$

\section{Dealing with missing data}

When analysing continuous data, if the SD was not reported, we estimated the mean and variance from the reported median, range, and sample size. ${ }^{32}$ When the SD and range were not available, the variance was estimated from the $\mathrm{p}$ value in the $t$ test. ${ }^{29}$

\section{Assessment of heterogeneity}

We will use $\chi^{2}$ and $\mathrm{I}^{2}$ statistics to examine statistical heterogeneity. The level of significance for the $\chi^{2}$ test is set at $0.10{ }^{33}{ }^{34}$ We will also estimate the between-study variance using the $\tau^{2}$ statistic. $^{29}$

\section{Assessment of reporting biases}

We will investigate potential small study biases using a funnel plot visually. ${ }^{35}$ In interpreting funnel plots, we will examine different possible reasons for funnel plot asymmetry and will relate these to results of this review. If more than 10 trials are included for a meta-analysis, we will perform statistical tests to investigate funnel plot asymmetry and will follow the recommendations stated in section 10.4 of the Cochrane Handbook for Systematic 
Reviews of Interventions. ${ }^{35}$ To assess selective outcome reporting, we will check trial protocols against published reports, if the protocol is available.

\section{Data synthesis}

When pooling is considered clinically significant, and data are enough, we will perform a random-effects meta-analysis with the Mantel-Haenszel statistical method for primary and secondary outcomes due to expected clinical heterogeneity across included RCTs and non-RCTs. ${ }^{36}$ When it is not appropriate to pool data, we will summarise the data for each trial in tabular forms. A forest plot was applied for the summary of results. Review Manager V.5.3 was used for meta-analysis. ${ }^{37}$

\section{Subgroup analysis and investigation of heterogeneity}

When the numbers of trials are sufficient, we will perform a subgroup analysis to explore the following potential sources of heterogeneity: ${ }^{38}$

1. Small tear versus large tear (different severity of disease).

2. RCTs versus studies with a non-RCT design (different study design).

We will compare the magnitude of treatment effects between the subgroups by inspecting overlapping CIs of summary estimates. Non-overlapping of CIs indicates a significant difference between subgroups.

\section{Assessment of confidence in estimates of effects}

Two reviewers will independently use the GRADE methodology to assess the quality of synthetic evidence for each outcome. ${ }^{31}$ The disagreements will be solved by discussion. If the arguments still exist after discussion, the third author will adjudge. Quality of evidence is rated 'very low', 'low', 'moderate' or 'high'. ${ }^{39}$ This method judges the overall quality of evidence for each outcome by considering the risk in the following domains including overall risk of bias, ${ }^{40}$ imprecision, ${ }^{41}$ inconsistency, ${ }^{42}$ indirectness ${ }^{43}$ and likelihood of publication bias. ${ }^{44}$ We will present the summary of the results of this review in a summary of findings table with the judgement of the overall quality of evidence for each synthetic outcome.

\section{Ethics and dissemination}

Ethical approval is not necessary for this protocol of systematic review since it does not involve any individual patient data and any privacy concerns. This protocol is reported according to the guidance of the Preferred Reporting Items for Systematic Reviews and Meta-Analysis Protocols (PRISMA-P) guideline ${ }^{45}$ We will publish this review by the PRISMA statement. ${ }^{46}$ Findings of this review will be disseminated as conference presentations and in the form of manuscripts submitted to Science citation index (SCI)-indexed peer-review journals.

\section{Patient and public involvement}

Patients or public were not involved in this study.

\section{DISCUSSION}

Our review will assess the available evidence for BMS during arthroscopic rotator cuff repair surgery in adults, provide estimates of the benefits of interventions and the associated harms, and evaluate the overall quality of the synthetic evidence using the GRADE approach. ${ }^{47}$ We will draw our conclusions based only on the findings from the quantitative or narrative synthesis in this review. Our results may assist orthopaedic surgeons in making clinical decisions regarding the optimal surgical technique of arthroscopic rotator cuff repair surgery and may guide researchers in prioritising future research and will outline remaining uncertainties in this area.

Contributors L-TK and JCY conceived the idea for this systematic review. L-TK, C-LC, P-AY, W-HH, C-CC and JCY developed the methodology for the systematic review protocol. The manuscript was drafted by L-TK and C-LC and revised by W-HH, C-CC, JCY. Y-ST developed the search strategy. L-TK and P-AY will screen potential studies, perform duplicate independent data extraction, risk of bias assessment, GRADE assessment. C-LC will conduct the data synthesis. C-CC with act as a third reviewer and arbitrator if necessary. C-CC is the guarantor of the review. All authors read and approved the final manuscript.

Funding The authors have not declared a specific grant for this research from any funding agency in the public, commercial or not-for-profit sectors.

Competing interests None declared.

Patient consent Not required.

Provenance and peer review Not commissioned; externally peer reviewed. Data sharing statement No additional data from this study are available.

Open access This is an open access article distributed in accordance with the Creative Commons Attribution Non Commercial (CC BY-NC 4.0) license, which permits others to distribute, remix, adapt, build upon this work non-commercially, and license their derivative works on different terms, provided the original work is properly cited, appropriate credit is given, any changes made indicated, and the use is non-commercial. See: http://creativecommons.org/licenses/by-nc/4.0/.

\section{REFERENCES}

1. Huang R, Wang S, Wang Y, et al. Systematic Review of AllArthroscopic Versus Mini-Open Repair of Rotator Cuff Tears: A MetaAnalysis. Sci Rep 2016;6:22857.

2. Osti L, Papalia R, Paganelli M, et al. Arthroscopic vs mini-open rotator cuff repair. A quality of life impairment study. Int Orthop 2010;34:389-94.

3. Duquin TR, Buyea C, Bisson LJ. Which method of rotator cuff repair leads to the highest rate of structural healing? A systematic review. Am J Sports Med 2010;38:835-41.

4. Mall NA, Tanaka MJ, Choi LS, et al. Factors affecting rotator cuff healing. J Bone Joint Surg Am 2014;96:778-88.

5. Hein J, Reilly JM, Chae J, et al. Retear Rates After Arthroscopic Single-Row, Double-Row, and Suture Bridge Rotator Cuff Repair at a Minimum of 1 Year of Imaging Follow-up: A Systematic Review. Arthroscopy 2015;31:2274-81.

6. Lafosse L, Brozska R, Toussaint B, et al. The outcome and structural integrity of arthroscopic rotator cuff repair with use of the double-row suture anchor technique. J Bone Joint Surg Am 2007;89:1533-41.

7. Prasathaporn N, Kuptniratsaikul S, Kongrukgreatiyos K. Single-row repair versus double-row repair of full-thickness rotator cuff tears. Arthroscopy 2011;27:978-85.

8. Sugaya $\mathrm{H}$, Maeda K, Matsuki K, et al. Repair integrity and functional outcome after arthroscopic double-row rotator cuff repair. A prospective outcome study. J Bone Joint Surg Am 2007;89:953-60.

9. Montgomery SR, Petrigliano FA, Gamradt SC. Biologic augmentation of rotator cuff repair. Curr Rev Musculoskelet Med 2011;4:221-30.

10. Saltzman BM, Jain A, Campbell KA, et al. Does the Use of PlateletRich Plasma at the Time of Surgery Improve Clinical Outcomes in Arthroscopic Rotator Cuff Repair When Compared With Control Cohorts? A Systematic Review of Meta-analyses. Arthroscopy 2016;32:906-18. 
11. Warth RJ, Dornan GJ, James EW, et al. Clinical and structural outcomes after arthroscopic repair of full-thickness rotator cuff tears with and without platelet-rich product supplementation: a metaanalysis and meta-regression. Arthroscopy 2015;31:306-20.

12. Hurley ET, Lim Fat D, Moran CJ, et al. The Efficacy of Platelet-Rich Plasma and Platelet-Rich Fibrin in Arthroscopic Rotator Cuff Repair: A Meta-analysis of Randomized Controlled Trials. Am J Sports Med 2018:036354651775139 (Published Online First: 21 February 2018).

13. Chuckpaiwong B, Berkson EM, Theodore GH. Microfracture for osteochondral lesions of the ankle: outcome analysis and outcome predictors of 105 cases. Arthroscopy 2008;24:106-12.

14. Steadman JR, Briggs KK, Rodrigo JJ, et al. Outcomes of microfracture for traumatic chondral defects of the knee: average 11-year follow-up. Arthroscopy 2003;19:477-84.

15. Jo $\mathrm{CH}$, Yoon $\mathrm{KS}$, Lee JH, et al. The effect of multiple channeling on the structural integrity of repaired rotator cuff. Knee Surg Sports Traumatol Arthrosc 2011;19:2098-107.

16. Milano G, Saccomanno MF, Careri S, et al. Efficacy of marrowstimulating technique in arthroscopic rotator cuff repair: a prospective randomized study. Arthroscopy 2013;29:802-10.

17. Osti L, Del Buono A, Maffulli N. Microfractures at the rotator cuff footprint: a randomised controlled study. Int Orthop 2013;37:2165-71.

18. Kida Y, Morihara T, Matsuda K, et al. Bone marrow-derived cells from the footprint infiltrate into the repaired rotator cuff. $J$ Shoulder Elbow Surg 2013;22:197-205.

19. Bilsel K, Yildiz F, Kapicioglu M, et al. Efficacy of bone marrowstimulating technique in rotator cuff repair. $J$ Shoulder Elbow Surg 2017:26:1360-6.

20. Sugaya H, Maeda K, Matsuki K, et al. Functional and structural outcome after arthroscopic full-thickness rotator cuff repair: singlerow versus dual-row fixation. Arthroscopy 2005;21:1307-16.

21. Boileau P, Brassart N, Watkinson DJ, et al. Arthroscopic repair of fullthickness tears of the supraspinatus: does the tendon really heal? J Bone Joint Surg Am 2005;87:1229-40.

22. Richards RR, An KN, Bigliani LU, et al. A standardized method for the assessment of shoulder function. J Shoulder Elbow Surg 1994;3:347-52.

23. Breckenridge JD, McAuley JH. Shoulder Pain and Disability Index (SPADI). J Physiother 2011;57:197.

24. Amstutz HC, Sew Hoy AL, Clarke IC. UCLA anatomic total shoulder arthroplasty. Clin Orthop Relat Res 1981:155:7-20.

25. Constant CR, Murley AH. A clinical method of functional assessment of the shoulder. Clin Orthop Relat Res 1987:160-4.

26. Neer CS, Watson KC, Stanton FJ. Recent experience in total shoulder replacement. J Bone Joint Surg Am 1982;64:319-37.

27. Hudak PL, Amadio PC, Bombardier C. Development of an upper extremity outcome measure: the DASH (disabilities of the arm, shoulder and hand) [corrected]. The Upper Extremity Collaborative Group (UECG). Am J Ind Med 1996;29:602-8.

28. Higgins JP, Altman DG, Gøtzsche PC, et al. The Cochrane Collaboration's tool for assessing risk of bias in randomised trials. BMJ 2011;343:d5928.
29. Higgins JPT, Green S. Cochrane handbook for systematic review of intervention version 5.1.0: The Cochrane Collaboration, 2011. (accessed Jun 2017).

30. Sterne JA, Hernán MA, Reeves BC, et al. ROBINS-I: a tool for assessing risk of bias in non-randomised studies of interventions. BMJ 2016;355:i4919.

31. Balshem $\mathrm{H}$, Helfand $\mathrm{M}$, Schünemann $\mathrm{HJ}$, et al. GRADE guidelines: 3. Rating the quality of evidence. J Clin Epidemiol 2011;64:401-6.

32. Hozo SP, Djulbegovic B, Hozo I. Estimating the mean and variance from the median, range, and the size of a sample. BMC Med Res Methodol 2005;5:13.

33. Higgins JP, Thompson SG. Quantifying heterogeneity in a metaanalysis. Stat Med 2002;21:1539-58.

34. Higgins JP, Thompson SG, Deeks JJ, et al. Measuring inconsistency in meta-analyses. BMJ 2003;327:557-60.

35. Sterne JAC, Egger M, D.eds M. Chapter 10: Addressing reporting biases. In: Higgins JPT, Green S, eds. Cochrane Handbook for Systematic Reviews of Intervention. Version 5.1.0: The Cochrane Collaboration, 2011

36. DerSimonian R, Laird N. Meta-analysis in clinical trials. Control Clin Trials 1986;7:177-88.

37. The Nordic Cochrane Centre, The Cochrane Collaboration. Review Manager (RevMan). 5.3. Copenhagen: The Nordic Cochrane Centre, The Cochrane Collaboration, 2011.

38. Higgins JP, Thompson SG. Controlling the risk of spurious findings from meta-regression. Stat Med 2004;23:1663-82.

39. Guyatt GH, Oxman AD, Vist GE, et al. GRADE: an emerging consensus on rating quality of evidence and strength of recommendations. BMJ 2008;336:924-6.

40. Guyatt GH, Oxman AD, Vist G, et al. GRADE guidelines: 4. Rating the quality of evidence-study limitations (risk of bias). J Clin Epidemiol 2011;64:407-15.

41. Guyatt GH, Oxman AD, Kunz R, et al. GRADE guidelines 6. Rating the quality of evidence-imprecision. J Clin Epidemiol 2011;64:1283-93.

42. Guyatt GH, Oxman AD, Kunz R, et al. GRADE guidelines: 7. Rating the quality of evidence-inconsistency. J Clin Epidemiol 2011;64:1294-302.

43. Guyatt GH, Oxman AD, Kunz R, et al. GRADE guidelines: 8 . Rating the quality of evidence-indirectness. J Clin Epidemiol 2011:64:1303-10.

44. Guyatt GH, Oxman AD, Montori V, et al. GRADE guidelines: 5 . Rating the quality of evidence-publication bias. J Clin Epidemiol 2011;64:1277-82.

45. Moher D, Shamseer L, Clarke M, et al. Preferred reporting items for systematic review and meta-analysis protocols (PRISMA-P) 2015 statement. Syst Rev 2015;4:1.

46. Moher D, Liberati A, Tetzlaff J, et al. Preferred reporting items for systematic reviews and meta-analyses: the PRISMA statement. PLoS Med 2009;6:e1000097.

47. Guyatt GH, Oxman AD, Kunz R, et al. Going from evidence to recommendations. BMJ 2008;336:1049-51. 